\title{
Microbial characteristics of the DMFT index in a high HIV prevalence setting
}

Dunstan Kalanzi ( $\sim$ dkalanzi@yahoo.com )

Makerere University https://orcid.org/0000-0003-0076-8573

\section{Harriet Mayanja Kizza}

Makerere University

\section{Damalie Nakanjako}

Makerere University College of Health Sciences

\section{Gerald Mboowa}

Makerere University College of Health Sciences

\section{Muhammad Mbabali}

Makerere University College of Health Sciences

\section{Edgar Kigozi}

Makerere University College of Health Sciences

\section{Fred Ashaba}

Makerere University College of Health Sciences

Ivan Sserwadda

Makerere University College of Health Sciences

\section{David Patrick Kateete}

Makerere University College of Health Sciences

\section{Beatrice Acan}

Makerere University College of Health Sciences

\section{Nelson K Sewankambo}

Makerere University College of Health Sciences

\section{Adrian Muwonge}

The University of Edinburgh The Roslin Institute

\section{Research}

Keywords: Human Immunodeficiency Virus, Network analysis, Dental caries, Periodontal disease, Oral microbiota, DMFT index

Posted Date: May 1st, 2020

DOl: https://doi.org/10.21203/rs.3.rs-25139/v1 
License: (c) (i) This work is licensed under a Creative Commons Attribution 4.0 International License. Read Full License 


\section{Abstract}

Background: Oral disease pathogenesis is primarily driven by microbial dysbiosis although diagnosis is routinely macroscopic. To improve early detection especially in HIV patients who are disproportionately affected, there is need to reconcile macroscopic and microscopic characteristics of disease. This study aimed to use amplicon sequencing data to characterize oral microbiota changes along the decayed, missing, filled teeth (DMFT) index.

Methods: Amplicon sequencing of the V6-V8 region of the 16S rRNA gene was done on DNA recovered from whole unstimulated saliva of 59 HIV positive and 29 HIV negative individuals, respectively. The microbial structure, composition and co-occurrence networks were characterized using QIIME-2, Phyloseq, Microbiome-1.9.2 and Metacoder in R.

Results: From a total of 2.6 million high quality sequence reads obtained, we characterized the oral microbiota into 2,093 operational taxonomic units (OTUs), 21 phyla and 239 genera. While oral microbiota did not cluster participants into distinct groups that track with the DMFT index, we observed the following: a) A steady increase in accessory microbiota while the core size ( $50 \%$ of richness) remained stable. b) The abundance of core genera such as Stomatobaculum, Peptostreptococcus and Campylobacter increased at onset of pathology (low DMFT), c) A general increase in oral microbial biomass with a typical log difference between gingivitis and periodontitis. $d$ ) The onset of pathology (low DMFT) was associated with massive reduction in oral microbial entropy.

Conclusions: Although oral microbial shifts along the DMFT index are not distinct, we have demonstrated the potential utility of microbiota dynamics to inform oral disease characteristics. We therefore propose a framework to inform future clinical oral metagenomic studies especially among HIV positive persons in resource limited settings.

\section{Introduction}

Oral diseases such as dental caries (tooth decay) and periodontal disease (gum disease) affect a third of the world's population ${ }^{1}$ costing in excess of half a trillion dollars ${ }^{2}$. HIV/AIDS predisposes patients to a higher risk of developing dental caries and periodontal disease compared to the general population 3,4 . In Uganda for example, 1.3 million people are infected with HIV, approximately $7.3 \%$ of the general population ${ }^{5}$ among who we have recently estimated $83.7 \%$ prevalence of dental caries ${ }^{6}$. On average this study population had a low DMFT index of 5.9. The DMFT index is a simple and common macroscopic tool that is used in epidemiological surveys of dental health status based on the number of decayed, missing (due to caries) and filled teeth ${ }^{7}$.

At present, the diagnosis of oral diseases is based on simple macroscopic characterization ${ }^{8}$ although the pathogenesis includes microbial effects. Indeed, some studies have attributed oral opportunistic infections, chronic conditions and immunogical variables to oral microbial dysbiosis due to HIV 
infection $^{9,10}$, but robust tracking of microbial pathobiological changes along the DMFT index has limited information. A variety of methods have been used to characterize the microbial composition of oral pathology, but due to the fastidious growth requirements of many of the resident oral bacteria, it is unlikely that they were successfully isolated by the conventional laboratory culture methods ${ }^{11}$. However, with the advent of the rapid and sensitive next-generation sequencing (NGS) technology, it is now possible to unpick the most intricate relationships in microbial communities ${ }^{12}$. Significantly, in developed countries, clinical metagenomics NGS (mNGS) is one of the fastest growing areas of medicine but little has been done in developing countries ${ }^{13}$. So, the utility of oral microbiota in characterizing oral diseases remains largely unexplored in countries with a high HIV burden, yet such knowledge would not only catalyze the development of innovative ways of improving management and diagnostics, but also guide the use of probiotic formulations in addition to antiretroviral therapy (ART).

Therefore, in this study we used mNGS to characterize oral microbiota shifts along the DMFT index in a high HIV burden setting in a developing country.

\section{Methods}

\section{Study participants and setting}

This was a cross sectional study carried out at Mulago Immune Suppression Syndrome (ISS) clinic (HIV care clinic) under the Makerere University Joint AIDS Program (MJAP). We recruited and sampled HIV negative and HIV positive adults at this clinic. The Mulago ISS clinic has been operating for over 15 years and provides HIV related services to approximately 16,000 patients $80 \%$ of who are on anti-retroviral treatment (ART). The clinicians treat on average 300 people every day, which makes it one of few areas to examine HIV related co-morbidities like dental disease.

\section{Sample size determination}

It is noteworthy that there are very few studies on oral clinical metagenomics in LMICs, therefore information on powering of such studies is still limited ${ }^{14}$. Since this was study was exploratory in nature, a sample size that maximizes the total number of genera detected (richness) was critical. Previous studies conducted elsewhere have used $50-65$ samples to detect up to $80 \%$ of genera ${ }^{15,16}$. Here we conveniently sampled 88 persons of the 168 who participated in this study between January and May 2018.

\section{Sample collection}

Saliva samples were collected between 9:00 AM and 12:00 noon to minimize the circadian effect on flow rate according to a published protocol ${ }^{17}$. For measurement of flow rate, saliva was collected for 5 minutes without any stimulation. Participants were asked not to swallow, but to expel the accumulated saliva into a calibrated plastic centrifuge tube at intervals over a period of 5 minutes. The volume of saliva estimated from the tube calibration (in $\mathrm{ml}$ ) was divided by 5 to give the flow rate $(\mathrm{ml} / \mathrm{min}$ ). Saliva 
samples were collected on ice, transported on ice and stored at $-80^{\circ} \mathrm{C}$ prior to component analysis. Oral examinations were performed by trained clinicians using dental probes and a mirror under suitable artificial light after saliva collection during the same visit.

\section{Clinical characterization of dental caries}

Caries was assessed using the World Health Organization (WHO) Radke's caries classification criteria and reported using the decayed (D), missing (M), filled (F), teeth (DMFT) index ${ }^{18}$. A DMFT score of $>0$ was considered as dental caries and thereafter, we categorized the participants into healthy (DMFT=0), low (DMFT=1-3), medium (DMFT=4-6), high (DMFT=7-13) and extremely high (DMFT>14).

\section{Clinical characterization of periodontal disease (gingivitis and periodontitis)}

Periodontal health was assessed using the Basic Periodontal Examination (BPE) ${ }^{19}$. This index integrates gingival inflammation, presence of calculus or overhanging margins and pocket depth to determine a particular score for a given sextant. All teeth present in a given sextant excluding the third molars were probed using a WHO BPE probe, which has a ball end $0.5 \mathrm{~mm}$ in diameter and a black band from 3.5 to $5.5 \mathrm{~mm}$, and the deepest pocket noted. Factoring in presence or absence of bleeding, calculus or over hangs and pocket depth, a score of zero to four was recorded for each sextant. The scores ranged between 1 and 4, which were categorized as gingivitis (BPE 1 and 2) and periodontitis (BPE of 3 and 4).

\section{Saliva processing and DNA extraction}

Saliva samples were processed as follows prior to DNA extraction using GenoLyse method as published by Bruker (USA). While in biosafety cabinet, 3 milliliters of saliva were transferred to $15 \mathrm{ml}$ sterile centrifuge tubes and an equal volume of $1 \% \mathrm{w} / \mathrm{v} \mathrm{NaCl}$ was added. The samples were vortexed vigorously for 1 minute until the specimen was fully liquefied. This was done in order to digest saliva to release the bacteria. Phosphate buffered saline (PBS) pH 6.8 was then added up to the $15 \mathrm{ml}$ mark. The specimens were then centrifuged at $3000 \mathrm{xg}$ for 15 minutes at $4^{\circ} \mathrm{C}$. The supernatant was then discarded, the resulting pellet was suspended into $1 \mathrm{ml}$ of 1 XPBS and then transferred to a $1.5 \mathrm{ml}$ nuclease free centrifuge tube. These were then centrifuged at $10000 \mathrm{x} g$ for 15 minutes prior to extraction of DNA using the GenoLyse method, following manufacturer's recommendations (Bruker, USA). Briefly, the supernatant was discarded and the pellet resuspended in 100mls of GenoLyse lysis buffer (A-LYS) prior to gentle vortexing to lyse the cells of the microorganisms including bacteria. Additional cell lysis was achieved by incubating the tubes at $95^{\circ} \mathrm{C}$ for 5 minutes prior to adding $100 \mathrm{mls}$ of GenoLyse neutralizing buffer (A-NB) to stop the action of A-LYS. The mixture was vortexed again for about 2 seconds prior to centrifugation at 13000 rpm for 5 minutes. DNA was collected from the supernatant and stored prior to use in subsequent analyses.

\section{S rRNA gene sequencing}


Aliquots of $30 \mu \mathrm{l}$ were then shipped under controlled ambient condition to Dalhousie University Integrated Microbiome Resource (IMR, Canada) ${ }^{20}$. Following purification of the amplicon pools using AMPure beads, sequencing of the V6-V8 16S rRNA variable loops was performed on the Illumina MiSeq platform (San Diego, CA, USA) using the 400 paired-end MiSeq run according to an established protocol ${ }^{21}$. Following sequencing, demultiplexed samples were returned.

\section{Sequence analysis}

A total of 88 paired end sequences in fastq format were received via an html file transfer link from the Dalhousie University Integrated Microbiome Resource $(\mathrm{IMR})^{20}$. Demultiplexed reads from the sequencing facility were then imported into the Qiime 2 pipeline ${ }^{22}$ for analysis. As part of quality control the pairedended reads were trimmed and merged into single ended reads. A QIIME artifact was then generated from these sequences and the metadata file. After dereplication, chimera-removal and denoising using DADA $2^{22}$ and OTUs dataset were generated. We filtered OTUs with a sequence depth of at least 3000 , retaining $90 \%$ of the samples and the corresponding metadata ${ }^{23}$.

\section{Microbial community structure}

Microbial diversity analysis was done using QIIME 2 and R-based packages; Phyloseq and Microbiome analyzer. The OTU-data output was then used to estimate the Alpha and Beta diversity indices. We considered observed Shannon for the Alpha diversity analysis ${ }^{24}$ while on the other hand, Beta diversity was estimated with Bray-Curtis, weighted and unweighted Unifrac distances ${ }^{22}$. To examine the association of clinical variables on the oral microbiota structure, we used a permutational multivariate analysis of variances (PERMANOVA) with the Adonis function (9,999 permutations) in phyloseq ${ }^{24}$ using the estimated Beta diversity indices as the outcome variable. The results were converted into a bar plot ranking the effect size $\mathrm{R}^{2}$ of each clinical variable and its statistical significance. To examine if patients can be clustered using beta diversity indices, we run a constrained distance-based redundancy using Bray-Curtis index as the beta diversity index. The analysis was constrained by DMFT and HIV status or periodontal disease type (gingivitis or periodontitis). We also run a principal coordinate analysis (PCoA) of weighted unifrac distance, which was used to estimate the total variance explained by first five components, henceforth referred to as TVE ${ }^{25}$ in Phyloseq. The impact of genera on TVE in the subsequent section was used to evaluate the most influential microbes in each DMFT category.

\section{Parameters for characterizing DMFT}

In order to map and track microbial changes along the DMFT index, we characterized changes in the alpha and beta diversity indices, abundance and prevalence, and co-occurrence dynamics.

\section{Microbial composition}

To investigate changes in abundance, we used Metacoder as described by Foster ${ }^{26}$ which combines phylogenetics and abundance. This allowed us to track changes of differentially abundant genera along 
the DMFT index. Briefly, this analysis was done using a Metacoder object generated from QIIME 2 taxonomic classification of OTUs generated before a naïve Bayes classifier trained on the most recent SILVA database at $97 \%$ similarity 27 . First, a training dataset was extracted using the primers used for sequencing our samples. The resultant database subset was then used to train the classifier for taxonomically assigning the OTUs. The heat tree highlights branches based on abundance. To determine the influential genera in DMFT category, we ranked the fifty most abundant genera, and then examined their impact on TVE by sequentially removing them from the data set representing each DMFT category.

\section{Core, and accessory microbiota}

To understand core and accessory changes as a proportion of the total richness along the DMFT index, first we defined the core, as the OTUs present in $85 \%$ of the samples at each DMFT category. Secondly, the pan-microbiota as the total number of unique OTUs in each of these categories, and the accessory as the difference between the pan and core microbiota in each category. We then analyzed the data to detect these microbial components in our samples using microbiome package 1.9. $2^{28}$, the output was summarized and plotted using Tidyrverse 1.2.1 and ggplot2 2.3.1 in R, respectively. To track the core as a proportion of microbial richness, we selected genera shared by all participants in all the DMFT categories then divided that by the richness at each stage. To examine the influence of genera, we first ranked nodes (genera) in this co-occurrence network by their centrality degree after which, we selected the top 50 genera (see co-occurrence network) and then examined their impact on TVE. This was done by sequentially dropping one genus from the select genera and compute the change TVE. The change in variance was visualized using bar plot in ggplot colored by the oxygen utilization capacity of each genus. It should be noted that we used the term invaders interchangeably with accessory microbiota, as they represented transient genera.

We also used published literature elsewhere to create two other categories i.e. oral-disease associated genera and normal flora ${ }^{29,30}$. (see Additional file 6: Supplementary DB variance analysis)

\section{Microbial co-occurrence networks}

We used the mean abundance correlation matrix of the genera in each DMFT category to map the changes in the co-occurrence network ${ }^{31,32}$. The "associate" function within the microbiome package version 1.9.2 in $\mathrm{R}$ (v3.5.1) was used to generate a genus-level spearman correlation matrix, here we set the FDR adjusted p-value at $0.05^{33}$ and pruned the matrix using a correlation coefficient of $>0.5$ and $<-0.5$. The resultant matrix was then converted into a directed-network object from which communities were extracted and visualized in igraph package version 1.2.2. The edges were colored based on statistical significance.

\section{A quasi-Poisson logistic regression for the DMFT}

To determine the factors associated with changes in biomass along the DMFT we develop a Poisson regression model in GLM where the outcome variable was mean taxonomic abundance (microbial 
biomass) using the Ime4 package in R version (v3.5.1). Data was split into five subsets each representing a DMFT category, we then run five separate models with same explanatory variables. It is these models we compared to examine the difference in estimates, the differences here represented the changes at each stage accounting for gender, HIV status and microbes at family level. Model comparison is done using sjtools in in R (v3.5.1).

\section{Results}

\section{Participants descriptive summary}

This study involved 38 and 50 male and female participants $(\mathrm{N}=88)$ with an average age of 39.5 years. The proportion of HIV positive participants was 67\% (59/88) with a median CD4 T cell count of 402 cells $/ \mathrm{mm}^{3}$, and a viral load of $\leq 50 \mathrm{copies} / \mathrm{mL}$ of blood. Most of the HIV positive participants were on ART. The mean salivary flow rate was $0.9 \mathrm{ml} / \mathrm{min}$ with no discernable difference between HIV positive and HIV negative participants. We observed a difference in the mean DMFT among HIV negative and positive participants of 5.9 and 4.9 , respectively (Table 1 ).

\section{Oral microbial community structure}

A total of 2,601,254 high quality $16 S$ rRNA sequences were recovered from the 88 participants. Apparently-healthy individuals on average generated 35,527 sequences while those with detectable oral pathology (DMFT>0) generated 32,515 sequences. Samples with the highest and lowest sequence count came from HIV negative and HIV positive participants, respectively (Additional file 1: Fig. S1). When the sequences were filtered to a depth of 3000, we retained 80 participants (Additional file 1: Fig. S1) from whom 2093 OTUs were generated with a median frequency of 14,351. There was no statistically significant difference in DMFT categories and HIV status regarding alpha diversity indices (Fig. 1A) but a statistically significant association ( $p>0.05$ ) was observed between DMFT and beta diversity indices such as the unweighted Unifrac and Bray-Curtis distances (Fig. 1B, Additional file 2: S2). There was some clustering along the DMFT index specifically separating low and medium categories with the ability to cluster the participants by HIV status but not periodontal disease (Fig. 1C, D).

\section{Oral microbial composition}

The above association was further explored at a taxonomic level i.e. core and accessory microbiota prevalence and abundance (Fig. 2A, B). The 2093 OTUs clustered into 21 phyla and 239 genera. Firmicutes (33.3\%), Bacteroidetes (32.3\%) and Proteobacteria (16\%) were the dominant phyla, however, at genus level Prevotella (12\%), Porphyromonas (3.7\%), Leptotrichia (3.4\%), Selenomonas (3.4\%) were the most predominant (Additional file 3: Fig. S3). Interestingly we detected genus Mycobacterium DNA from three HIV positive patients and a potential linear relation with CD4 T cell count (Additional file 4: Fig. S4).

\section{Apparently healthy individuals (DMFT $=0$ )}


Among the eleven apparently healthy individuals the core and pan microbiota size was 65 and 86 genera respectively, i.e. an accessory microbiota of 21 genera. The most abundant core genera included; Ruminococcus, Mogibacterium, Megasphaera, Campylobacter, Atopobium and Actinomyces (Fig. 2B). The core, normal flora and genera associated with oral pathology accounted for $49 \%, 23 \%$ and $27 \%$ respectively while the accessory microbiota accounted for $1 \%$ (Fig. 3). Of the 65 genera observed among apparently healthy individuals, 49 were shared across the different DMFT categories, hence forth referred to as the DMFT core.

\section{Low DMFT}

The transition from apparently healthy to low DMFT category was characterized by a slight increase in the proportion of the accessory microbiota but the proportion of the core at this stage remained relatively unchanged. This was associated with an increase in the abundance of Stomatobaculum, Peptostreptococcus and a reduction in Atopobium and Actinomyces (Fig. 2B). The DMFT microbial model suggests a significant difference in microbial biomass between participants with concurrent gingivitis and periodontitis. Here the families with significant changes in abundance include Weeksellacae, Veillonellaceae, Streptococaccae, Ruminoccocaea among others (Fig. 3). We also noted that clusters 2 \& 5 (Fig. 4A, B), were exclusively composed of HIV positive participants, the former was characterized by a low abundance of genera that are associated with oral disease and the latter by those that are part of the core.

\section{Medium and High DMFT}

The medium DMFT category was distinctly separated by microbial clustering (Fig. 1C, D) and also characterized by a slight increase in the proportion of the accessory microbiota. The core, normal flora and oral disease associated genera accounted for oral microbial richness of $48 \%, 22 \%$ and $27 \%$ respectively (Fig. 3). This change was associated with an enrichment of Ruminococcus, Peptospteptococcus and Lautropia (Fig. 2B). Participants in the high DMFT category with concurrent periodontitis carried significantly more microbial biomass than those with concurrent gingivitis (Additional file 5: Fig. S5). Indeed, at this stage the accessory microbes accounted for $9 \%$ of the microbiota in the oral cavity (Fig. 4C). However, families whose abundance significantly changed primarily belong to the core; Carnobacteriaceae, Neisseriaceae, Micrococcaceae and Streptococcaceae (Fig. 5B).

\section{Extremely high DMFT}

In comparison with the apparently healthy and low DMFT categories, this stage was characterized by a slight increase in Solobacterium, Oribacterium, Neisseria, Granulicatella, Atopobium, Abiotrophia and a reduction in Campylobacter (Fig. 2A). On the other hand, the proportion of the accessory microbiota fell at this stage (Fig. 4C).

\section{Microbial co-occurrence network remodeling along the DMFT index}




\section{Changes in community entropy}

We characterized remodeling of the co-occurrence networks based on the DMFT categories as shown in Fig. 6B. There was a characteristic change in entropy i.e. the proportion of genus pairwise association that are statistically significant (Fig. 6A). In apparently-healthy patients, the four main communities were dominated by core microbiota. In addition, the co-occurrence network of apparently healthy patients was characterized by a high level of entropy as only a few pair-wise correlations were statistically significant $(\mathrm{p}<0.05)$. These included Treponema, Johnsonella and Rikenellaceae, Porphyromonas, Alloprevotella and Aggregatibacter, Tannerella, Fusobacterium and Gemella, Stomatobaculum, Veillonella and Solobacterium, Campylobacteria and Oribacterium. The low DMFT category was associated with a massive reduction of entropy i.e. 99\% of pairwise correlations were statistically significant i.e. a change from random to non-random or a dramatic reduction in entropy (Fig. 6A). We also noted that although the communities include members of the core, most of the genera belonged to the accessory community. As the severity of dental caries progresses, entropy returns i.e. co-occurrence clusters gradually change from non-random to random.

\section{Genera associated changes in variance}

We noted among the apparently healthy participants a variance change of up to $5 \%$, most of which was attributable to core genera (Fig. $6 \mathrm{C}$ ). This suggests that the most influential genera at this stage belong to core (see full list of genera identified as influential in Additional file 6: Supplementary DB variance analysis). In the low DMFT category, the variance changes were up to $10 \%$, attributable to 20 genera, most of which belonged to the accessory oral microbiota. These included; Treponema, Desulfoplanes, Desulfosporosinus, Sphaerochaeta, Arenimonas, and Microbacterium among others. Notably these belonged to the families with statistically significant shifts in abundance at this stage. The medium and high DMFT categories were also characterized by changes in variance of as high as $10 \%$ and we observed that the composition of influential genera changed from aerobic to anaerobic. Unlike the low DMFT category, here $98 \%$ of the influential genera belonged to the core (see Additional file 6: Supplementary DB variance analysis). The extremely high DMFT category was characterized by limited change in variance, and here too almost all the influential genera are members of the core.

\section{Discussion}

The pathogenesis and progression of dental caries has for long been linked to a microbial precursor ${ }^{8}$ but $^{-}$ its routine diagnosis in developing countries is almost exclusively macroscopic i.e. based on the DMFT index ${ }^{7}$. This is mainly because microbial culture-based testing is laborious and complicated ${ }^{11}$, which makes comprehensive and timely delivery of clinical results prohibitively protracted. However, with the advent of culture-independent NGS approaches like 16S rRNA amplicon sequencing ${ }^{34}$, we can narrow this gap in the developing countries where the HIV epidemic may be disproportionately driving the incidence of oral disease. In this study, we used amplicon sequencing data to characterize changes in oral microbiota along the routinely used diagnostic DMFT index. 
To the best of our knowledge, this is the first study in this setting that has characterized oral microbiota along the DMFT index. On average, the oral microbiota was characterized using 32,515 sequences, generating 2093 OTUs that clustered into 21 phyla and 239 genera. We observed no significant differences in alpha diversity along the DMFT index. Since structure and composition of the oral microbiota can be influenced by parameters such as saliva flow rate ${ }^{35}$, we accounted for this by establishing the flow rate (mean=0.9 $\mathrm{ml} / \mathrm{min}$ ) among the participants and compared it to the microbial structure. We observed clustering by HIV status with or without considering other factors such as gender and age at this microbial structural level.

Microbiota is integral to the oral cavity and plays a critical role in maintaining its integrity ${ }^{8}$. In apparentlyhealthy individuals this is expected to manifest as stability in structure and composition ${ }^{11,36}$. In this study, we observed a relatively stable core size along the DMFT index. The proportion of the core relative to the rest of the microbiota was incredibly stable with 49 genera present across the DMFT categories. Indeed, the notion of the microbiome core is commonly investigated as a proxy for resident microbes of a given microbiome ${ }^{37}$. In addition to the 49 core membership, we characterised a core for each DMFT category. Among apparently healthy individuals, we observed genera such as Streptococcus, Staphylococcus, Corynebacterium, Veillonella, Granulicatella and Gemella, most of which have been previously reported as normal flora of the oral cavity ${ }^{38}$. The proportions of genera characterised as normal flora, core and that which are associated with oral pathology were relatively low. The proportion of accessory microbiota, also referred to as invaders was at its lowest among the apparently healthy individuals. This observation supports the notion that resident genera dominate the oral cavity by inhibiting invaders through the production of substances such as fatty acids, peroxides and bacteriocins ${ }^{39}$. This concerted activity has also been characterised among functional communities ${ }^{40}$, which in this study are analogous to co-occurrence communities. Indeed, among healthy individuals we observed that the co-occurrence communities are dominated by members of the core such as Johnsonella, Rikenella, Porphyromonas, Alloprevotella Tannerella, Fusobacterium and others. This further supports the notion that resident bacteria maintain the integrity of the oral cavity among healthy individuals ${ }^{39}$. The organisms observed were predominantly anaerobic ${ }^{29}$ probably due to microbial succession that occurs during the formation of dental plaque ${ }^{41}$.

Onset of pathology is widely associated with oral microbiome dysbiosis ${ }^{8}$. While there is ambiguity on what defines dysbiosis, there is common agreement on the nature of forces that modulate the onset of pathology ${ }^{11}$. The forces are similar to selection forces i.e. changes in fitness, growth and reproduction of microbes manifested as fluxes in prevalence and abundance ${ }^{8}$. In the low DMFT category we observed that amidst such forces, the core size still remains stable with a slight increase in the proportion of accessory microbes. The increase in the proportion of invaders was associated with presence of anaerobic lactate fermenters like Sharpea, Lawsonella and Olsenella, previously implicated in the onset of endodontic infections and acute apical abscesses ${ }^{42,43}$. While the size of the core does not change, there was considerable increase in abundance of genera such as Stomatobaculum, Peptostreptococus, 
Campylobacter and Mogibacterium which have been associated with onset of subgingival pathology 44 . In this category, we noted that individuals with concurrent gingivitis carried significantly more microbes than those with concurrent periodontitis. In this regard, families such as Weeksellacae, Veillonellaceae, Streptococcaceae and Ruminococcacaea accounted for most of the change in abundance. This is most probably due changes in the local environment and microbial interactions favoring the growth of these organisms that have been associated with dental caries ${ }^{29,45}$ and labial abscess ${ }^{46}$. We also noted the absence of genera such as Roseburia, which is associated with health elsewhere ${ }^{47}$, its absence here probably supports the onset of pathology. The onset of pathology can be viewed as an onset of a constraint to the normal microbial community interaction. In this study, we showed that this constraint is detectable as a change in entropy, in this case $99 \%$ of the pairwise association were statistically significant. In other words, the probability that the associations observed are occurring randomly is very low, which suggests introduction of an overwhelming constraint. This change in entropy is also associated with an increase (up to 10\%) in variation attributable to genera such as Lachnospira, Treponema, Aggregatibacter, Corynebacteria and Bifidobacteria whose roles in pathogenesis of oral diseases are well documented ${ }^{48-51}$. However, some of the influential genera such as Ferruginibacter and Sphaerochaeta are part of the accessory microbiota.

The proportion of the accessory microbiota in the medium and high DMFT categories increased compared to the apparently healthy and low DMFT categories. Here too the proportion of core remained stable and comparatively similar to normal flora and oral disease associated genera. In literature, the onset of pathology is associated with interaction of different microorganisms in a dynamic and concerted polymicrobial synergy to form a cariogenic biofilm within which the community changes as caries progress from early onset (initial demineralization) to deeper lesions with dentin exposure ${ }^{52}$. In particular there is an increase in abundance of core members, Rothia and Mogibacterium, which are associated with dental caries ${ }^{53}$ and periodontal disease ${ }^{53,54}$, and Lautropia, which has been isolated from oral cavities of HIV infected children ${ }^{55}$ but is not associated with clinical oral disease ${ }^{55,56}$. However, as severity of dental caries progresses, the resident microbes appear to be overwhelmed, seen here as a surge in the accessory microbiota. Microbiota at this point appears to have gone through significant remodeling because most of the invading population in the medium category then become established as members of the core. Moreover, it is in these two categories and at low DMFT that we observed the highest proportion of the least biologically characterized organisms.

Although the extremely high DMFT category is characterized based on six individuals, nonetheless, we observed a generalized increase in abundance of the members although the core and accessory size is smaller. The core was dominated by genera such as Peptostreptoccus, Mogibacterium and Atopobium which have been implicated in chronic oral pathology ${ }^{57}$. We also observed a massive reduction in variation and genera such as Solobacterium, Ruminococcus, Neisseria, Campylobacter, Atopobium and Abitrophia have the largest change in abundance. 
From these findings, we propose a microbial model of the DMFT index as a foundation for clinical metagenomics in LMIC settings. In addition to presence and abundance of genera, we used microbial structure and taxonomic characteristics. We specifically characterized genus level influence based on their impact on variance and most importantly shift in entropy. To this effect, the findings here suggest the following; a) Microbial structure; alpha diversity index does not discriminate along the DMFT index but beta diversity does. b) Taxonomic composition; the DMFT core genera approximate normal flora in most categories of the DMFT index other than extremely high DMFT and the proportion of accessory microbiota (invading bacteria) increases with dental caries. In addition, at the onset of pathology/low DMFT, the number of genera whose abundance significantly changes belong to the core, this gradually changes to the accessory in the medium and high DMFT categories. c) Entropy; the onset of pathology creates a microbiome wide constraint detectable as a change in entropy. Almost all genera-pairwise correlations are statistically significant at this stage interpreted as a reduction in microbial entropy. d) Concurrent gingivitis versus periodontitis; we noted a significant difference in microbial abundance i.e. the low DMFT category best represents gingivitis while the high DMFT category represents most cases of periodontitis. Most importantly we observed that when we account for clinical and microbiota characteristics, there is a significant difference between HIV positive and negative participants. With this information, a testable hypothesis about pathobiology, probiotic supplementation and treatment can be generated and tested to improve dental clinical practice in the management of people living with HIV.

It is important to note that in this study, we did not have equal numbers of patients in each group i.e. 11, $32,21,16$ and 8 for healthy, low, medium, high and extremely high DMFT categories. This has the potential of increasing the level of uncertainty for the estimates made for groups with fewer individuals. However, we observed a considerable level of consistency in microbes within groups and therefore the impact of sample size per group is likely to be limited.

\section{Conclusions}

In this study, we characterized oral microbiota dynamics along the DMFT index and although the microbial characteristics did not offer a categorical output of the DMFT index, they provided the following insights; a) the onset of pathology/low DMFT category appears to be driven by significant changes in core and accessory genera abundance, $b$ ) the medium and high DMFT categories were associated with a steady increase in invading/accessory microbiota likely responsible for biomass distinction between gingivitis and periodontitis, $\mathrm{c}$ ) the influential genera in apparently healthy patients are predominantly core members but this changes to accessory in medium and high DMFT categories, $d$ ) onset of pathology was associated with a massive reduction in oral microbial entropy. Therefore, using this information we propose a microbial framework for characterizing the DMFT index as a foundation for improving diagnostics and management of oral disease in HIV positive participants in resource limited settings.

\section{Declarations}

\section{Ethical considerations}


The study was approved by the School of Medicine Research and Ethics committee of Makerere University (\#REC REF 2017-053), and it was conducted in accordance with the ethical standards outlined in the 1964 Declaration of Helsinki and its later amendments. Written informed consent was obtained from all participants prior to their participation in the study.

\section{Consent for publication}

Not applicable

\section{Availability of data and materials}

The sequences generated for this study have been deposited in the Sequence Read Archive of NCBI (Project Number: PRJNA627249). All other data is contained in the main manuscript and supplemental files.

\section{Competing interests}

The authors declare that they have no competing interests

\section{Funding}

This work is part of a research fellowship supported by Grant Number D43TW010132 supported by Office of The Director, National Institutes of Health (OD), National Institute of Dental \& Craniofacial Research (NIDCR), National Institute of Neurological Disorders and Stroke (NINDS), National Heart, Lung, And Blood Institute (NHLBI), Fogarty International Center (FIC), National Institute on Minority Health and Health Disparities (NIMHD). Its contents are solely the responsibility of the authors and do not necessarily represent the official views of the supporting office.

\section{Authors' contributions}

DK, HMK, DN, DPK, EK and FA generated the study concept and design. DK and MM acquired and organized the data. EK and FA performed the experiments. AM analyzed the data, AM, GM, IS, DK and DPK interpreted the data. DK, AM and GM wrote the first draft of the manuscript. HMK, DN, DPK, EK, FA, BA, AM, MM, NS contributed critical revisions and important intellectual content to the manuscript. All authors read and approved the final manuscript.

\section{Acknowledgements}

We are gratefully to the participants who took part in this study.

\section{Author information}

Not applicable 


\section{References}

1 Frencken, J. E. et al. Global epidemiology of dental caries and severe periodontitis - a comprehensive review. Journal of clinical periodontology 44 Suppl 18, S94-s105, doi:10.1111/jcpe.12677 (2017).

2 Righolt, A. J., Jevdjevic, M., Marcenes, W. \& Listl, S. Global-, Regional-, and Country-Level Economic Impacts of Dental Diseases in 2015. J Dent Res 97, 501-507, doi:10.1177/0022034517750572 (2018).

3 Cavasin Filho, J. C. \& Giovani, E. M. Xerostomy, dental caries and periodontal disease in HIV+ patients. The Brazilian journal of infectious diseases : an official publication of the Brazilian Society of Infectious Diseases 13, 13-17 (2009).

4 Malhotra, A., Ahlawat, J., Hegde, M. \& Mahajan, A. Dental caries status in human immunodeficiency virus-positive and acquired immunodeficiency syndrome patients. Indian Journal of Oral Sciences 7, 103106, doi:10.4103/0976-6944.194235 (2016).

5 Uganda AIDS Commission. The Uganda HIV and AIDS Country Progress Report July 2016-June 2017. (2017).

6 Kalanzi, D. et al. Prevalence and factors associated with dental caries in patients attending an HIV care clinic in Uganda: a cross sectional study. BMC Oral Health 19, 159, doi:10.1186/s12903-019-0847-9 (2019).

7 Anaise, J. Z. Measurement of dental caries experience--modification of the DMFT index. Community dentistry and oral epidemiology 12, 43-46 (1984).

8 Proctor, D. et al. Microbial biogeography and ecology of the mouth and implications for periodontal diseases. bioRxiv, 541052, doi:10.1101/541052 (2019).

9 Lewy, T. et al. Oral Microbiome in HIV-Infected Women: Shifts in the Abundance of Pathogenic and Beneficial Bacteria Are Associated with Aging, HIV Load, CD4 Count, and Antiretroviral Therapy. AIDS Res Hum Retroviruses 35, 276-286, doi:10.1089/aid.2017.0200 (2019).

10 Mukherjee, P. K. et al. Dysbiosis in the oral bacterial and fungal microbiome of HIV-infected subjects is associated with clinical and immunologic variables of HIV infection. PloS one 13, e0200285, doi:10.1371/journal.pone.0200285 (2018).

11 Kilian, M. et al. The oral microbiome - an update for oral healthcare professionals. Br Dent $J$ 221, 657666, doi:10.1038/sj.bdj.2016.865 (2016).

12 Morgan, X. C. \& Huttenhower, C. Chapter 12: Human microbiome analysis. PLoS computational biology 8, e1002808, doi:10.1371/journal.pcbi.1002808 (2012). 
13 Prifti, E. J. D. Z. The new science of metagenomics and the challenges of its use in both developed and developing countries. (Springer, 2015).

14 Xian, P. et al. The Oral Microbiome Bank of China. International Journal of Oral Science 10, 16, doi:10.1038/s41368-018-0018-x (2018).

15 Charlson, E. S. et al. Disordered microbial communities in the upper respiratory tract of cigarette smokers. PloS one 5, e15216, doi:10.1371/journal.pone.0015216 (2010).

$16 \mathrm{Kirst}, \mathrm{M}$. E. et al. Dysbiosis and alterations in predicted functions of the subgingival microbiome in chronic periodontitis. Applied and environmental microbiology 81, 783-793, doi:10.1128/aem.02712-14 (2015).

17 Navazesh, M. \& Kumar, S. K. Measuring salivary flow: challenges and opportunities. Journal of the American Dental Association (1939) 139 (5 suppl), 35s-40s (2008).

18 Petersen Poul Erik , B. R. J., World Health Organization. Oral Health Surveys Basic Methods 5th Edition. (2013).

19 Periodontology, B. S. o. Basic Periodontal Examination. (2011).

20 Centre for Comparative Genomics and Evolutionary Bioinformatics (CGEB) - Dalhousie University. Integrated Microbiome Resource (IMR). (2014+).

21 Comeau, A. M., Douglas, G. M. \& Langille, M. G. Microbiome Helper: a Custom and Streamlined Workflow for Microbiome Research. mSystems 2, doi:10.1128/mSystems.00127-16 (2017).

22 Bolyen, E. et al. QIIME 2: Reproducible, interactive, scalable, and extensible microbiome data science. PeerJ Preprints 6, e27295v27292, doi:10.7287/peerj.preprints.27295v2 (2018).

23 Durbin, R., Eddy, S., Krogh, A. and Mitchison, G. Biological sequence analysis., (Cambridge Univ. Press., 1990).

24 McMurdie, P. J. \& Holmes, S. phyloseq: An R Package for Reproducible Interactive Analysis and Graphics of Microbiome Census Data. PloS one 8, e61217, doi:10.1371/journal.pone.0061217 (2013).

25 Gower, J. C. Some distance properties of latent root and vector methods used in multivariate analysis. Biometrika 53, 325-338 (1966).

26 Foster, Z. S. L., Sharpton, T. J. \& Grünwald, N. J. Metacoder: An R package for visualization and manipulation of community taxonomic diversity data. PLoS computational biology 13, e1005404, doi:10.1371/journal.pcbi.1005404 (2017).

27 Quast, C. et al. The SILVA ribosomal RNA gene database project: improved data processing and webbased tools. Nucleic acids research 41, D590-596, doi:10.1093/nar/gks1219 (2013). 
28 Leo, L. S., Shetty.; et al. . Introduction to the microbiome R package, <https://microbiome.github.io/> (2019).

29 Tanner, A. C., Kressirer, C. A. \& Faller, L. L. Understanding Caries From the Oral Microbiome Perspective. Journal of the California Dental Association 44, 437-446 (2016).

30 Diaz, P. I., Hoare, A. \& Hong, B. Y. Subgingival Microbiome Shifts and Community Dynamics in Periodontal Diseases. Journal of the California Dental Association 44, 421-435 (2016).

31 Mandakovic, D. et al. Structure and co-occurrence patterns in microbial communities under acute environmental stress reveal ecological factors fostering resilience. Scientific reports $\mathbf{8}, 5875$, doi:10.1038/s41598-018-23931-0 (2018).

32 Fernandez, M., Riveros, J. D., Campos, M., Mathee, K. \& Narasimhan, G. Microbial "social networks". BMC genomics 16, S6, doi:10.1186/1471-2164-16-S11-S6 (2015).

33 Bioconductor. Microbiome, <https://bioconductor.org/packages/devel/bioc/html/microbiome.html> (2019).

34 Zarco, M. F., Vess, T. J. \& Ginsburg, G. S. The oral microbiome in health and disease and the potential impact on personalized dental medicine. Oral diseases 18, 109-120, doi:10.1111/j.16010825.2011.01851.x (2012).

35 Proctor, D. M. et al. A spatial gradient of bacterial diversity in the human oral cavity shaped by salivary flow. Nature communications 9, 681, doi:10.1038/s41467-018-02900-1 (2018).

36 Marsh, P. D. \& Zaura, E. Dental biofilm: ecological interactions in health and disease. Journal of clinical periodontology 44 Suppl 18, S12-s22, doi:10.1111/jcpe.12679 (2017).

37 Aguirre de Carcer, D. The human gut pan-microbiome presents a compositional core formed by discrete phylogenetic units. Scientific reports 8, 14069, doi:10.1038/s41598-018-32221-8 (2018).

38 Aas, J. A., Paster, B. J., Stokes, L. N., Olsen, I. \& Dewhirst, F. E. Defining the normal bacterial flora of the oral cavity. Journal of clinical microbiology $43,5721-5732$, doi:10.1128/jcm.43.11.5721-5732.2005 (2005).

39 Devine, D. A., Marsh, P. D. \& Meade, J. Modulation of host responses by oral commensal bacteria. Journal of oral microbiology 7, 26941, doi:10.3402/jom.v7.26941 (2015).

40 Duran-Pinedo, A. E. \& Frias-Lopez, J. Beyond microbial community composition: functional activities of the oral microbiome in health and disease. Microbes and infection 17, 505-516, doi:10.1016/j.micinf.2015.03.014 (2015). 
41 Marsh, P. D. Are dental diseases examples of ecological catastrophes? Microbiology (Reading, England) 149, 279-294, doi:10.1099/mic.0.26082-0 (2003).

42 Siqueira, J. F. \& Rôças, I. N. Microbiology and Treatment of Acute Apical Abscesses. Clinical Microbiology Reviews 26, 255-273, doi:10.1128/cmr.00082-12 (2013).

43 Siqueira, J. F., Jr. \& Rocas, I. N. Uncultivated phylotypes and newly named species associated with primary and persistent endodontic infections. Journal of clinical microbiology 43, 3314-3319, doi:10.1128/jcm.43.7.3314-3319.2005 (2005).

44 Shi, M. et al. The Subgingival Microbiome of Periodontal Pockets With Different Probing Depths in Chronic and Aggressive Periodontitis: A Pilot Study. Front Cell Infect Microbio/ 8, 124, doi:10.3389/fcimb.2018.00124 (2018).

45 Jiang, Q., Liu, J., Chen, L., Gan, N. \& Yang, D. The Oral Microbiome in the Elderly With Dental Caries and Health. Front Cell Infect Microbio/ 8, 442, doi:10.3389/fcimb.2018.00442 (2018).

46 Slenker, A. K., Hess, B. D., Jungkind, D. L. \& DeSimone, J. A. Fatal Case of Weeksella virosa Sepsis. Journal of clinical microbiology 50, 4166-4167, doi:10.1128/jcm.01761-12 (2012).

47 Tamanai-Shacoori, Z. et al. Roseburia spp.: a marker of health? Future microbiology 12, 157-170, doi:10.2217/fmb-2016-0130 (2017).

48 Liu, F., Ma, R., Wang, Y. \& Zhang, L. The Clinical Importance of Campylobacter concisus and Other Human Hosted Campylobacter Species. Front Cell Infect Microbio/ 8, 243, doi:10.3389/fcimb.2018.00243 (2018).

49 Visser, M. B. \& Ellen, R. P. New insights into the emerging role of oral spirochaetes in periodontal disease. Clinical Microbiology and Infection 17, 502-512, doi:https://doi.org/10.1111/j.14690691.2011.03460.x (2011).

50 Hajishengallis, G., Darveau, R. P. \& Curtis, M. A. The keystone-pathogen hypothesis. Nature reviews. Microbiology 10, 717-725, doi:10.1038/nrmicro2873 (2012).

51 Mira, A., Simon-Soro, A. \& Curtis, M. A. Role of microbial communities in the pathogenesis of periodontal diseases and caries. Journal of clinical periodontology 44 Suppl 18, S23-s38, doi:10.1111/jcpe.12671 (2017).

52 Bowen, W. H., Burne, R. A., Wu, H. \& Koo, H. Oral Biofilms: Pathogens, Matrix, and Polymicrobial Interactions in Microenvironments. Trends in microbiology 26, 229-242, doi:10.1016/j.tim.2017.09.008 (2018).

53 Fridman, D., Chaudhry, A., Makaryus, J., Black, K. \& Makaryus, A. N. Rothia dentocariosa Endocarditis: An Especially Rare Case in a Previously Healthy Man. Texas Heart Institute journal 43, 255-257, 
doi:10.14503/thij-15-5068 (2016).

54 Casarin, R. C. et al. Detection of Mogibacterium timidum in subgingival biofilm of aggressive and nondiabetic and diabetic chronic periodontitis patients. Brazilian journal of microbiology : [publication of the Brazilian Society for Microbiology] 43, 931-937, doi:10.1590/s1517-838220120003000012 (2012).

55 Rossmann, S. N. et al. Isolation of Lautropia mirabilis from oral cavities of human immunodeficiency virus-infected children. Journal of clinical microbiology 36, 1756-1760 (1998).

56 Peterson, S. N. et al. The Dental Plaque Microbiome in Health and Disease. PloS one 8, e58487, doi:10.1371/journal.pone.0058487 (2013).

$57 \mathrm{Xu}$, L. et al. Dynamic Alterations in Salivary Microbiota Related to Dental Caries and Age in Preschool Children With Deciduous Dentition: A 2-Year Follow-Up Study. Frontiers in Physiology 9 , doi:10.3389/fphys.2018.00342 (2018).

\section{Table}

\section{Table 1: Descriptive Statistics of Samples}

\begin{tabular}{|c|c|c|c|}
\hline & \multirow[t]{3}{*}{ Number (\%) } & \multicolumn{2}{|c|}{ HIV status } \\
\hline & & HIV+ & HIV- \\
\hline & & $59(67.1)$ & $29(32.9)$ \\
\hline \multicolumn{4}{|l|}{ Sex } \\
\hline Female & $50(56.8)$ & $34(68.0)$ & $16(32.0)$ \\
\hline Male & $38(43.2)$ & $25(65.8)$ & $13(34.2)$ \\
\hline $\begin{array}{l}\text { Age in years: median } \\
\text { (IQR) }\end{array}$ & $34.5(27.5,44.0)$ & $38.2(12.2)$ & $33(24,42)$ \\
\hline \multicolumn{4}{|l|}{ HIV status } \\
\hline Negative & $29(32.9)$ & - & - \\
\hline Positive & $59(67.1)$ & - & - \\
\hline \multicolumn{4}{|l|}{ ART status in months } \\
\hline 1 & $30(34.1)$ & $30(50.8)$ & - \\
\hline 2 & $29(33.0)$ & $29(49.2)$ & - \\
\hline CD4 count: median (IQR) & $402(231,596)$ & $402(231,596)$ & - \\
\hline \multicolumn{4}{|l|}{ Viral load } \\
\hline$<50$ & $49(62.8)$ & $49(62.8)$ & - \\
\hline $50+$ & $10(100)$ & $10(100)$ & - \\
\hline DMFT: mean (SD) & $5.2(4.9)$ & $4.9(4.6)$ & $5.9(5.5)$ \\
\hline \multicolumn{4}{|l|}{ DMFT category } \\
\hline Healthy (0) & $11(12.5)$ & $8(72.7)$ & $3(27.3)$ \\
\hline Low (1-3) & $32(36.4)$ & $20(62.5)$ & $12(37.5)$ \\
\hline Medium (4-6) & $21(23.9)$ & $17(80.9)$ & $4(19.1)$ \\
\hline High (7-13) & $16(18.2)$ & $10(62.5)$ & $6(37.5)$ \\
\hline Extremely high $(\geq 14)$ & $8(9.0)$ & $4(50.0)$ & $4(50.0)$ \\
\hline \multicolumn{4}{|l|}{ Periodontal status } \\
\hline Gingivitis & $51(57.9)$ & $31(60.8)$ & $20(39.2)$ \\
\hline Periodontitis & $37(42.1)$ & $28(75.7)$ & $9(24.3)$ \\
\hline $\begin{array}{l}\text { Saliva flow rate: mean } \\
\text { (SD) }\end{array}$ & $0.9(0.5)$ & $0.8(0.5)$ & $1.0(0.5)$ \\
\hline
\end{tabular}


Figures
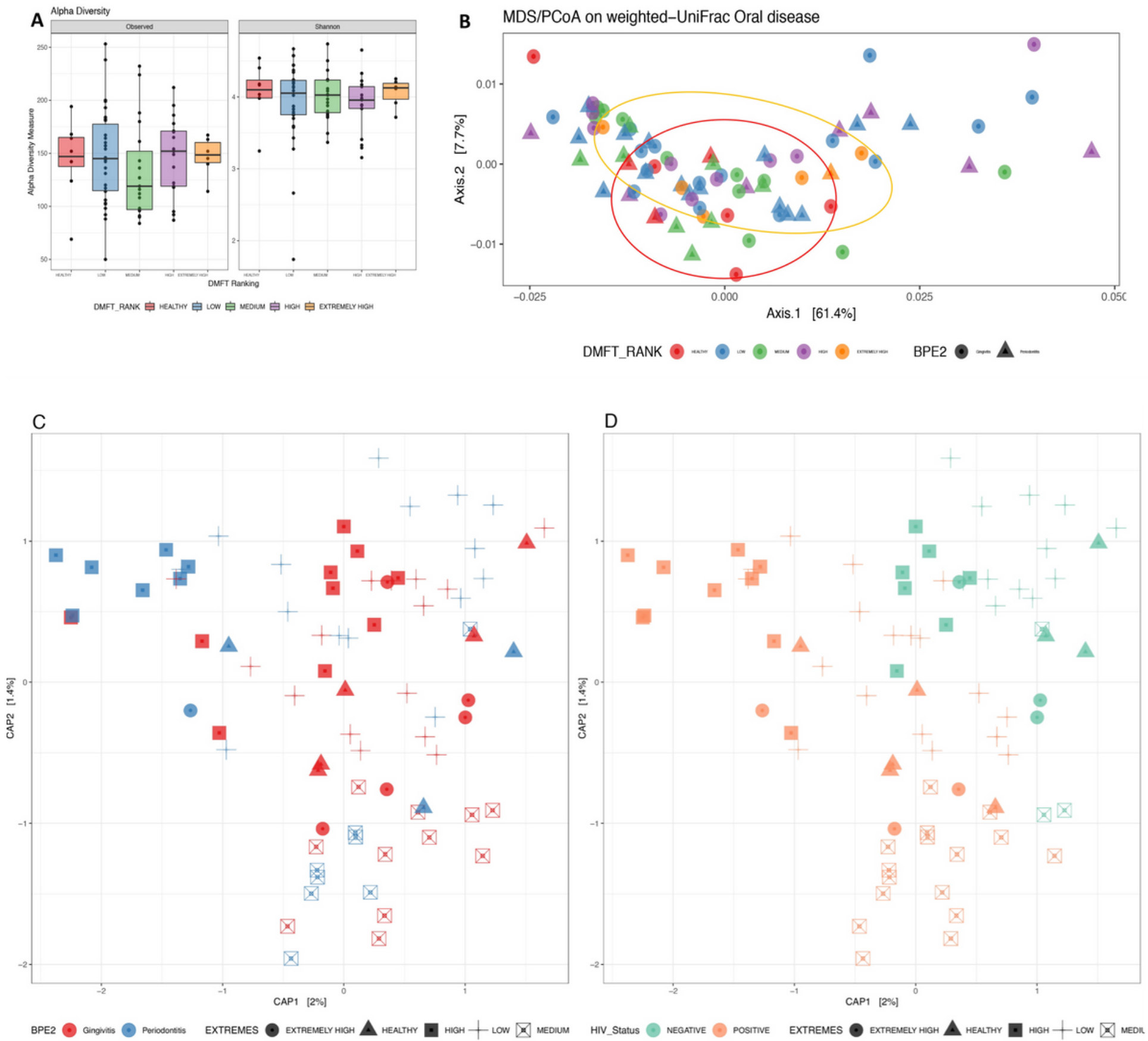

\section{Figure 1}

Attributes used to assess the oral microbial structure. Panel A shows the alpha diversity along the DMFT index, panel $B$ shows the beta diversity analysis using weighted unifrac along the DMFT index; here the red and yellow rings show the clustering of healthy and extremely-high DMFT categories, panels C and D show beta diversity using Bray Curtis distances for periodontal disease and HIV status with clustering of the low and medium DMFT categories evident. 


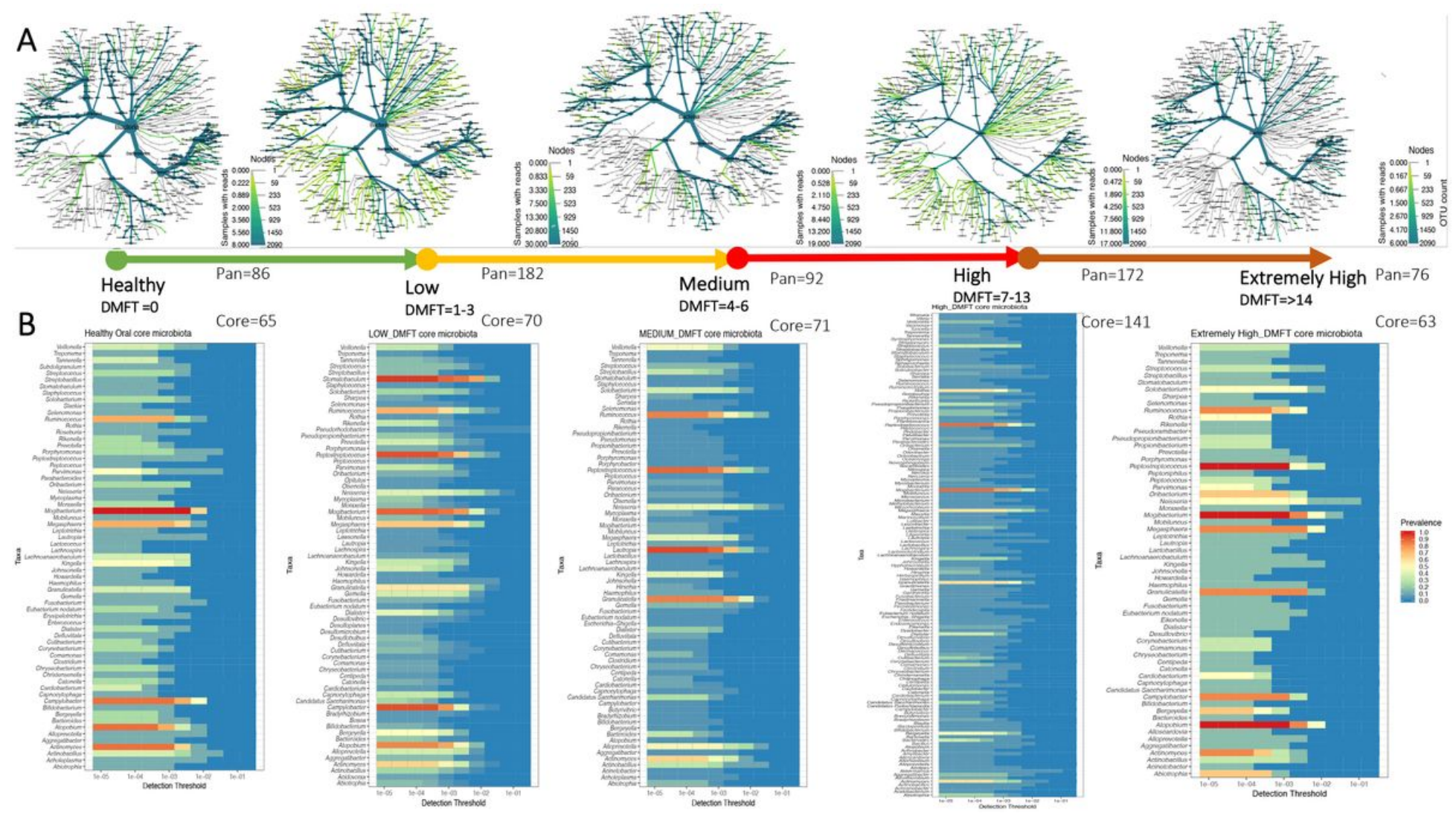

Figure 2

The pan $(A)$ and core $(B)$ oral microbiota tracked along the DMFT index in order of severity. Panel A and B represent genera abundance and prevalence respectively.

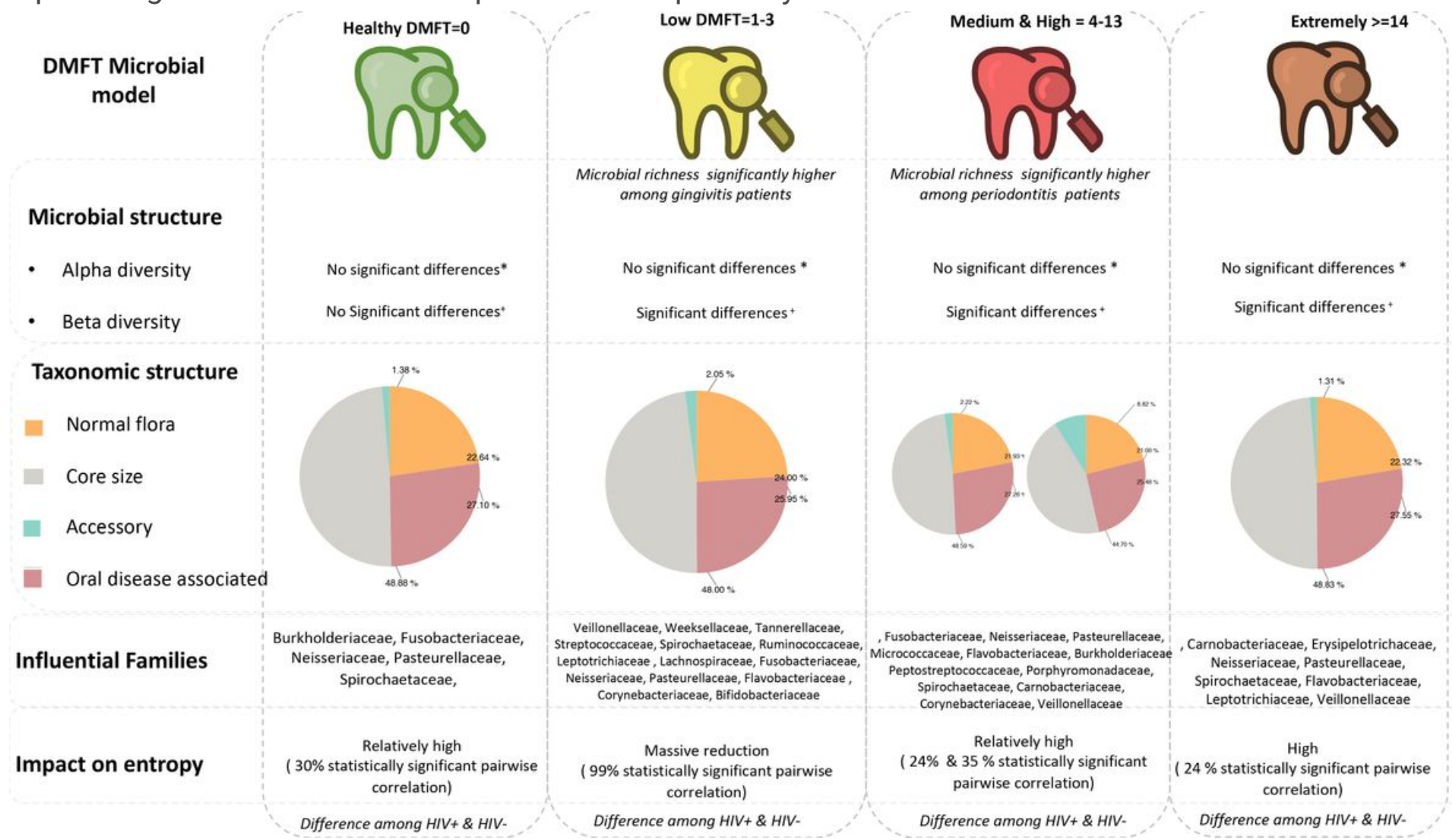




\section{Figure 3}

Shows our proposed clinical metagenomic model for DMFT in low and middle-income countries.
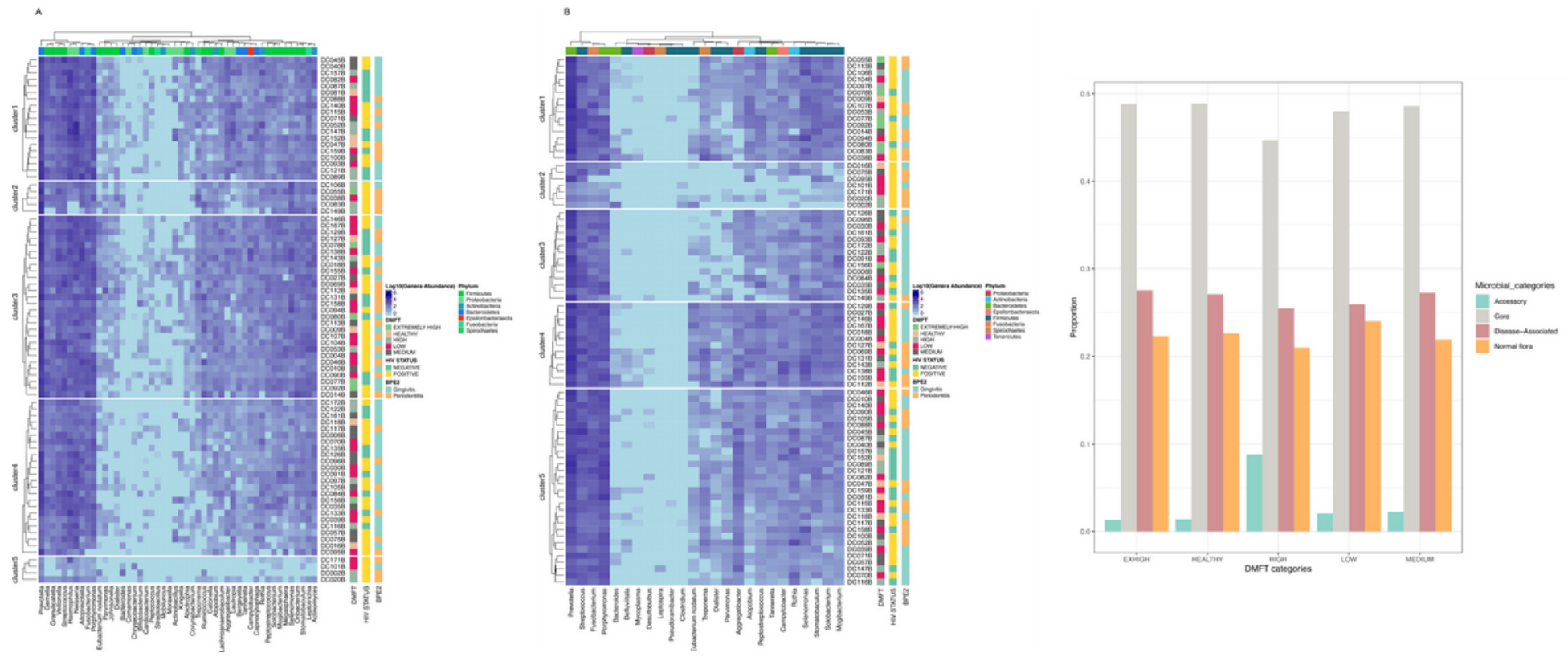

\section{Figure 4}

The changes of microbial components tracked along the DMFT index. Panel A shows the DMFT core, which is genera present at all stages of the DMFT characterized by sample ID, HIV status and periodontal disease, B shows genera associated with oral disease characterized by sample ID, HIV status and periodontal disease and $\mathrm{C}$ shows the changes in the proportions of microbial groups along the DMFT categories. 
A

hrssous $* *$

sex $*$

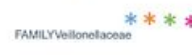

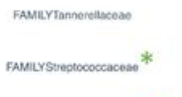

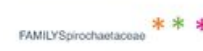

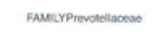

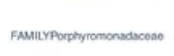

FAMIVYPeptostreptoosecascene

Farmer Pasteuretiocense

Faveromers
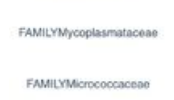

FAMerrechnoseracesese

$+2$

FAMTVEEngicelourchacose

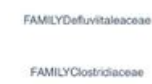

FAMLYCampitebacionacose

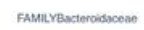

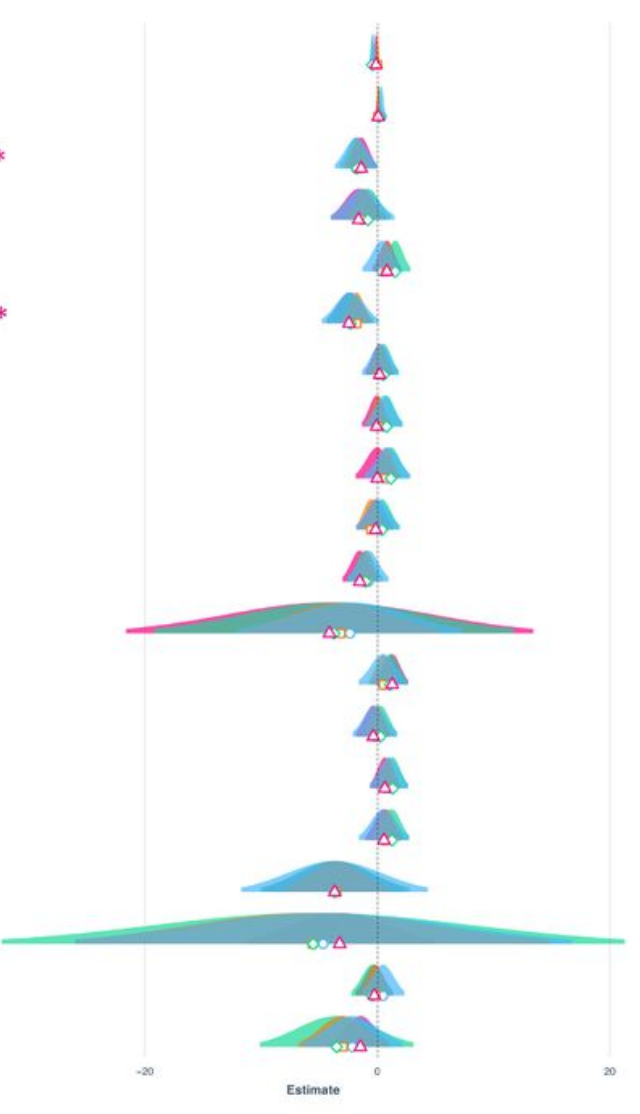

B

B

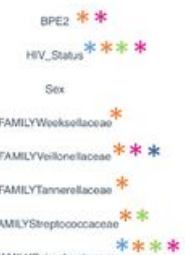

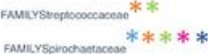

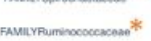

rescerprevonessona

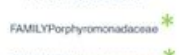

FAMILY Pestostroptocoscacacose *

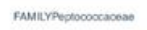

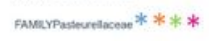

ravilucomers

Fanitrinoessoriacose $* * * *$

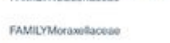

Faverimarococcacosse * *

Famitropototichissosose * * *

Faminnachooseracoso $* * *$

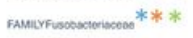

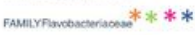

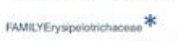

* * * *

FAMilyCarnstacteriscente $* *$

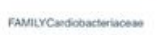

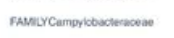

ramilveurathoideracose * *

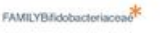

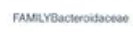

FAMrexalopociaceas

Fameraerocoscosoast

\section{Figure 5}

The Poisson regression model of taxonomic abundance and clinical attributes. Panels A \& B show a comparison of models generated from datasets that represents each stage of the DMFT. Note that for panel A we compare four models because the model for extremely high did not converge. 
A

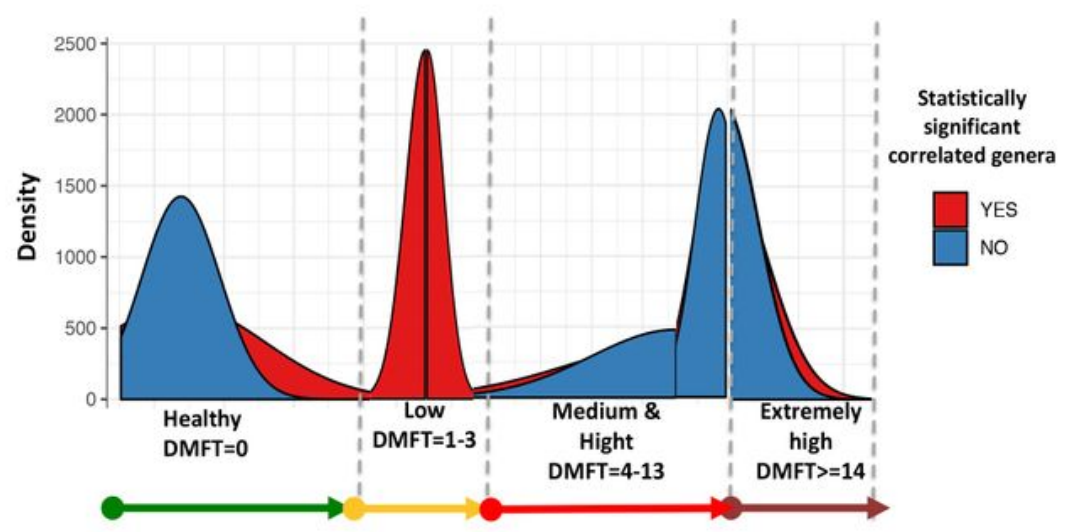

B

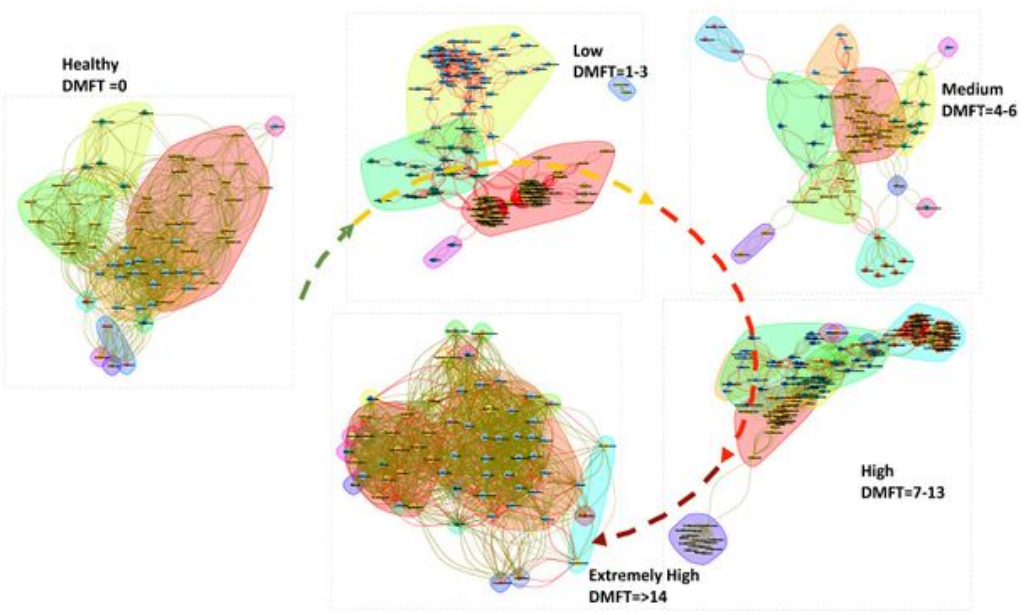

C

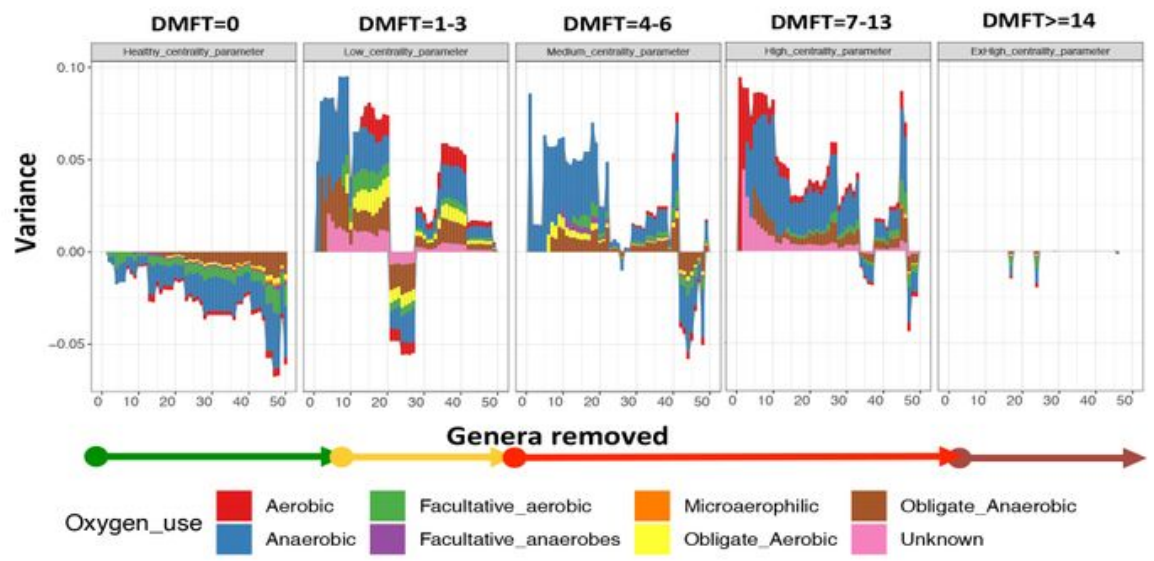

\section{Figure 6}

The oral microbial co-occurrence network characteristics. Panel A shows the changes in microbial community entropy, B shows the co- occurrence network remodeling and $\mathrm{C}$ shows variance changes and the genera to which the variance is attributable.

\section{Supplementary Files}


This is a list of supplementary files associated with this preprint. Click to download.

- AdditionalFiles.zip 